\title{
Púrpura trombocitopénica trombótica refractaria
}

\author{
Thrombotic thrombocytopenic purpura
}

\author{
Emanuel Araújo, Rita Boaventura, M Teresa Cardoso \\ Centro Hospitalar São João. Porto, Portugal
}

\begin{abstract}
Thrombotic thrombocytopenic purpura (TTP) is a rare immune hematologic disease, with an incidence of 1 to 13 per million person-years, in which activity of ADAMTS13 (a vWF-cleaving protease) is severely reduced. We present a case of a 62 years' woman with gastrointestinal and neurologic symptoms along with thrombocytopenia, renal failure and positive anti-ADAMTS13. Treated initialy with plasma apheresis and corticosteroids with good response, she relapsed after two weeks of treatment. Then Rituximab was used with success. In patients treated with plasma apheresis the risk of relapse is high. Newer therapies like Rituximab are effective as we report in this case.
\end{abstract}

Palabras clave: ADAMTS13. Plasmaféresis. Púrpura trombocitopénica trombótica. Rituximab

Keywords: ADAMTS13. Plasma apheresis. Rituximab. Thrombotic thrombocytopenic purpura

\section{Introduction}

Thrombotic thrombocytopenic purpura (TTP) is a rare hematologic disease, in which activity of ADAMTS13 (a vWFcleaving protease) is severely reduced. Diagnosis is based on clinical and laboratorial findings and without treatment the mortality reaches $90 \%$. After the advent of plasmapheresis, the mortality decreased, but in many cases, there is a risk of relapse. In that situation, immuno-modulation with rituximab can be an option. The combination of different therapeutics could lead to better results, as explained in the clinical case below. ${ }^{1}$

\section{Case Report}

A 62-year-old African woman, with no controlled hypertension on the last ten years, presented with epigastric diffuse pain for six days, nausea and vomits. She referred asthenia plus weight loss in the last month. It was prescribed metoclopramide and a proton pump inhibitor without improvement so she recurred to the hospital. On physical examination, she presented normal blood pressure and pulse as well as normal cardiac and pulmonary auscultation. Abdominal examination revealed diffuse pain without signs of peritoneal irritation. She had an episode of dizziness with tremors and slowed speech. She maintained sphincter control and didn't have loss of consciousness. Blood cell count revealed a platelet count of 10,000 with LDH 1451 IU/L, presence of schizocytes (4/5 per field) and acute renal failure with serum creatinine of $1,36 \mathrm{mg} /$ $\mathrm{dL}$, urea $85 \mathrm{mg} / \mathrm{dL}$ and calculated creatinine clearance of $48,6 \mathrm{~mL} /$ $\mathrm{min} / 1,73 \mathrm{~m}^{2}$ (CKD-EPI). Urine analysis revealed proteins, erythrocytes and leukocytes. Spleen was normal in abdominal ultrassound and abdominal and brain TC were normal. Bone marrow showed increased number of megakaryocites and blood cells precursors. Anti-ADAMTS13 was strongly positive and immunologic study was negative. She was admitted in intermediate care unit and started prednisolone $1 \mathrm{mg} / \mathrm{Kg} /$ day plus daily plasmapheresis. The renal function improved and the number of platelets increased to 160,000 after twelve sessions of plasmapheresis. Then, plasmapheresis sessions were reduced to each two days, but the disease relapsed after 3 sessions. Plasmapheresis was started daily again. Meanwhile, endoscopic study revealed chronic gastritis and
Helicobacter pylori infection. A protocol of eradication was initiated, successfully. Further causes of immune thrombocytopenia as neoplasia were investigated, by doing abdomino-pelvic CT, thyroid ultrassound, mammography, colonoscopy and cytology of cervix, all negative. After more 8 sessions of daily plasmapheresis with platelets decreasing and presence of schizocytes, it was decided to start rituximab after excluding viral infection or tuberculosis and administration of Influenzae and Streptococcus pneumonia vaccine. Rituximab was given in three sessions, two weeks of interval each one reaching a total dose of $2600 \mathrm{mg}$ combined with daily plasmapheresis. After the $2^{\text {nd }}$ administration of rituximab, number of platelets started to increase, LDH dropped and schizocytes were negative. Plasmapheresis was reduced to each two days, for more 4 sessions and stopped 3 days before last administration of rituximab. Although her improvement in the fiftieth after admission, she developed respiratory insufficiency and tachycardia. A lung CT scan was performed which revealed a bilateral pulmonary embolism. She initiated hypocoagulation with warfarine with improvement of symptoms. She was discharged from the hospital after two months of internment, without any symptoms, and a platelet count of 280,000 with a diagnosis of TTP. The presence of antiphospholipid syndrome is supported by venous thrombosis, although immunologic study was negative (anti-DsDNA, anti-cardiolipin antibodies and lupus anticoagulant). After eighteen months of follow-up consultations she had no symptoms, maintained normal platelets count, low LDH and normal creatinine.

\section{Discussion}

Thrombotic thrombocytopenic purpura (TTP) is one of the thrombotic microangiopathic (TMA) syndromes, with an estimated incidence of 1 to 13 per million person-years, caused by an autoimmune mechanism, although rare nonimmune inherited forms were described. ${ }^{1,2,3}$ Some factors like female sex, black ethnicity and obesity are considered risk factors for the disease. ${ }^{1,2}$ TTP remains a life-threatening disease with a mortality of 10-20\% even with correct therapeutic management. It's defined by clinical (multi-symptoms mainly involving the central nervous system and/or gastrointestinal system) and hematologic criteria (microangiopathic 
hemolytic anemia and severe thrombocytopenia).2,3 The mechanism below the disease involves a severe functional ADAMTS13 deficiency $(<5 \%)$ with formation of platelet-rich microthrombi within small arterioles. ${ }^{1}$ In $90 \%$ of cases, this deficiency is caused by the presence of anti-ADAMTS13 IgG antibodies that inhibit the proteolytic activity of ADAMTS13 toward VWF. In a minority of cases, anti-ADAMTS13 is not detectable so the disease can be explained by mutations in ADAMTS13.,4 The symptoms are related to the widespread of microthrombi and consequent ischemic alterations in brain $(60 \%)$, heart $(25 \%)$, gastrointestinal $(35 \%)$ and renal impairment with rarely acute renal failure. Blood analysis show microangiopathic hemolytic anemia and consumption thrombocytopenia, with high reticulocyte count and the presence of schizocytes. ${ }^{1,2}$ Approximately $10 \%$ of patients with TTP develop other antibodies either isolated or associated to symptoms of another autoimmune disease (mainly antidsDNA and SLE)." Other associated states include pregnancy, some infections like Helicobacter pylori or HIV, cancer and organ transplantation. ${ }^{1,5}$ The management of TTP has changed along years, but plasmapheresis and corticosteroids are the first line therapy for TTP. Before the use of plasmapheresis, mortality was approximately $90 \%{ }^{1,3,6}$ Initially, our patient responded to this therapy. Even though plasmapheresis removes vWF multimers and autoantibody inhibitors of ADAMTS13, the risk of relapse with plasmapheresis is high in $30-60 \%$ of patients, as occurred in our case after seventeen sessions of it. In case of relapse, immune modulation is an option and pulses of cyclophosphamide, cyclosporine or azathioprine were used with success. ${ }^{1,6}$ Splenectomy remain the last resource with $70 \%$ of success. However, the risk of infection is slightly higher comparing to non-splenectomized TTP patients. ${ }^{7}$ Promising therapeutics under investigation include $\mathrm{N}$-acetylcysteine, bortezomib, recombinant ADAMTS13 and caplacizumab, an inhibitor of the glycoprotein-Ib/IX-von Willebrand factor axis. ${ }^{8}$ Our patient had elevated levels of Anti-ADAMTS13, with a relapse after prednisolone and plasmapheresis. In this cases rituximab can be useful. Rituximab targets CD20 expressed in the surface of premature and mature B-cells, and has been used off-label for immune diseases, including TTP. Recent studies demonstrate $98 \%$ of effectiveness with standard protocol of $375 \mathrm{mg} / \mathrm{m}^{2}$, especially in patients with elevated levels of antiADAMTS13, like our case. Secondary effects are tolerable ${ }^{6}$ and they were not observed in this patient. Rituximab was a secure and effective treatment in refractory TTP in this case and eighteen months after treatment she remains with no symptoms. With this case, we reported that administration of rituximab improved platelet count in patient with refractory TTP, despite plasmapheresis and corticotherapy.

\section{References}

1. Joly BS, Coppo P, Veyradier A. Thrombotic thrombocytopenic purpura. Blood 2017;129(21):2836-46

2. George JN, Nester CM. Syndromes of thrombotic microangiopathy. N Engl J Med. 2014;371(7):654-66

3. Moake JL. Thrombotic microangiopathies. N Engl J Med. 2002;347(8):589-600.

4. Scully M. Inhibitory anti-ADAMTS 13 antibodies: measurement and clinical application. Blood Rev. 2010;24(1):11-6.

5. Kamangar F, Sheikhattari P, Mohebtash M. Helicobacter pylori and its effects on human health and disease. Arch Iran Med. 2011;14(3):192-9.

6. Tun NM, Villani GM. Efficacy of rituximab in acute refractory or chronic relapsing non-familial idiopathic thrombotic thrombocytopenic purpura: a systematic review with pooled data analysis. J Thromb Thrombolysis. 2012;34(3):347-59.

7. Kappers-Klunne MC, Wijermans P, Fijnheer R, Croockewit AJ, van der Holt B, de Wolf $\mathrm{JT}$, et al. Splenectomy for the treatment of thrombotic thrombocytopenic purpura. $\mathrm{Br}$ J Haematol. 2005;130(5):768-76.

8. Coppo P, French Reference Center for Thrombotic M. Treatment of autoimmune thrombotic thrombocytopenic purpura in the more severe forms. Transfus Apher Sci. 2017;56(1):52-6. 